\title{
The 10-word learning task in the differential diagnosis of early Alzheimer's disease and elderly depression: a cross sectional pilot study
}

Dierckx, E., Engelborghs, S., De Raedt, R., Van Buggenhout, M., De Deyn, P. P., D'Haenens, E., Verté, D., \& Ponjaert-Kristoffersen, I. (2011). The 10-word learning task in the differential diagnosis of early Alzheimer's disease and elderly depression: a cross sectional pilot study. Aging \& Mental Health, 15, 113-121. 


\begin{abstract}

\section{Objectives}

Identification of early Alzheimer's disease (AD) has become very important. Episodic memory tasks appear to have predictive power for indicating early AD. Deficits in encoding and storage processes that are characteristic of $\mathrm{AD}$ however must be distinguished from non-AD deficits that can also affect memory, including difficulties that may be present in depression. This pilot study was set up to ascertain whether a 10 word-list-learning task (delayed recognition and rate of forgetting) may be useful in making the differentiation between mild $\mathrm{AD}$ and depression.
\end{abstract}

\title{
Method
}

A Dutch version of Rey's Auditory Verbal Learning Test was administered to 36 mild AD patients, 41 depressed patients and 47 healthy controls. Data were analyzed cross sectional.

\section{Results}

ROC analyses showed that for differentiating mild AD and depression, both delayed recognition and percentage of forgetting have sufficient diagnostic accuracy.

\section{Conclusion}

Percentage of forgetting had the highest diagnostic accuracy for differentiating mild $\mathrm{AD}$ and depressed patients and may be useful in the early detection of AD.

Keywords: dementia; elderly depression; memory; recognition; delayed recall; serial verbal learning test 


\section{Introduction}

The development of pharmacological treatment options for Alzheimer's Disease (AD) that aim to modify the disease process increases the need for early diagnosis of AD (Swainson et al., 2001). Patients might benefit from cognition-enhancing or future disease-modifying drugs (Sonkusare, Kaul, \& Ramarao, 2005). Due to an unprecedented growth of scientific knowledge, Dubois et al. (2007) proposed revised diagnostic research criteria for $\mathrm{AD}$. These new criteria are centered on a clinical core of early and significant episodic memory impairment (together with supportive biomarker criteria). Episodic memory tasks appear to have predictive power for early $\mathrm{AD}$, as the episodic memory function is anatomically located within the medial temporal lobe structures, including the hippocampus. These structures contain the highest densities of AD neuropathological lesions. Most studies that have addressed the issue of early detection of AD have emphasized the diagnostic value of delayed free recall deficits. However, impaired delayed recall is not in itself evidence of an AD-related disorder. The genuine deficits in encoding and storage processes that are characteristic of $\mathrm{AD}$ must be distinguished from non-AD deficits that can also affect delayed recall, including difficulties that may be present in depression (Dubois et al., 2007).

This study was set up to ascertain whether a 10-word-list learning task (10-RAVLT) (a standardized Dutch variation of Rey's Auditory Verbal Learning Test) incorporated in a Dutch dementia battery (VVKP Werkgroep Klinische gerontopsychologie, 1998), may be useful in differentiating between mild AD and depression in elderly patients. The usefulness of the concepts of (1) recognition and (2) rate of forgetting will be studied in the light of early diagnosis. 
Generally speaking, memory processes can be divided into three different successive phases, namely (1) acquisition or encoding, (2) consolidation, and (3) retrieval (Tuokko \& Hadjistavropoulos, 1998).

Several studies (Perry, Watson, \& Hodges, 2000; Au, Chan, \& Chiu, 2003) have shown that the impairment of acquisition and, more specially, the consolidation of episodic memory traces are the earliest signs of the underlying degenerative process of $\mathrm{AD}$. This process originates in the medial temporal lobe, in particular in the entorhinal cortex and the hippocampus (Karrasch, Sinerva, Gronholm, Rinne, \& Laine, 2005). Therefore, assessment of encoding and consolidation may be useful by detecting subtle entorhinal or hippocampal dysfunctions.

\section{Recognition}

Delayed recall involves the retrieval of a previously encoded word, whereas delayed recognition measures only whether or not a word has been encoded and consolidated (Shankle et al., 2005). In contrast to patients with mild AD, who may fail to consolidate information effectively, depressed patients may demonstrate essentially normal memory consolidation (Hart, Kwentus, Taylor, \& Harkins, 1987). In general, it is assumed that depressed elderly patients show normal delayed recognition (Fossati et al., 2004). In summary, we assume that among AD patients, due to consolidation deficits, delayed recognition performance will be very poor, whereas depressed patients will show normal delayed recognition.

\section{Rate of forgetting}

AD patients fail to consolidate information effectively (Hart, Kwentus, Taylor, \& Harkins, 1987; Perry et al., 2000). Moreover, it has indeed been shown (Kopelman, 1992) that in AD patients information rapidly disappears from episodic memory, 
whereas in elderly depressed patients there is no retention deficit (Fossati et al., 2004; Bearden et al., 2006). The rate of forgetting paradigm may thus be clinically useful for distinguishing patients with early AD from elderly depressed patients (Hart, Kwentus, Taylor, \& Harkins, 1987). Forgetting can be defined as a decrease in performance over time represented by the slope of a line connecting the acquisition terminus to performance at follow-up (MacDonald, Stigsdotter-Neely, Derwinger, \& Backman, 2006). In most cases, delayed free recall has been used to operationalize this rate of forgetting. Because there is always a possibility that differences in forgetting rates result in part from poor initial learning rather than from differences in the ability to retain information over time, we argue that it might be better to operationalize the rate of forgetting not by means of delayed free recall, but by means of the percentage of forgetting in which the amount of initially learned information is taken into account (Estevez-Gonzalez, Kulisevsky, Boltes, Otermin, \& Garcia-Sanchez, 2003). Given the fact that $\mathrm{AD}$ patients have more difficulty retaining information than depressed elderly patients, one could argue that the percentage of forgetting will be higher in AD patients than in depressed patients.

\section{Aim and objectives}

In general, we wanted to ascertain whether the 10-RAVLT, which is a widely and frequently used verbal learning task, could be used in the early differentiation between mild AD and depression in elderly patients.

We hypothesized that (1) AD patients would obtain lower scores on delayed recognition, whereas depressed elderly patients would have normal recognition scores and that (2) unlike depressed patients, who are able to retain information over time, AD patients would show a higher percentage of forgetting. 


\section{Methods and measures}

The results of this cross-sectional study were obtained between March 2004 and May 2005.

\section{Participants}

The inclusion criteria of the study population were as follows: (1) a diagnosis of probable AD, according to the DSM-IV and NINCDS-ADRDA criteria (American Psychiatric Association, 1994; McKhann et al., 1984) or (2) a diagnosis of major depression, according to the DSM-IV criteria (American Psychiatric Association, 1994) established by the clinicians in the institutions (neurologists in the memory clinic and psychiatrists in the psychiatric institutions) following an elaborate workup consisting of a general physical and neurological examination, blood screening, structural neuroimaging (consisting of a computerized tomography scan of the brain and/or magnetic resonance imaging and functional brain imaging - single photon emission computed tomography (SPECT)), and an electroencephalogram, as well as an extensive neuropsychological examination consisting of tests other than those included in this study administered by the institutions' psychologists. Additionally, a comprehensive clinical interview was performed by the clinician to diagnose major depression. This procedure was carried out in the memory clinic and in the psychiatric institutions where most of the depressed patients were recruited.

AD patients who scored over 19 on the Mini Mental State Examination (MMSE) (Folstein, Folstein, \& McHugh, 1975) were classified as mildly demented. Because the differentiation between depression and $\mathrm{AD}$ is most difficult in the earliest stages of $\mathrm{AD}$, only mild $\mathrm{AD}$ patients were included in the analyses.

The exclusion criteria were the presence of a bipolar disorder or a psychotic depression and the presence of a delirium according to the Confusion Assessment Method (CAM) (Inouye et al., 1990). 
Allocation to the 2 patient groups was based on the diagnosis carried out by the clinicians in the institutions. When an AD patient appeared to have significant depressive symptoms, he/she was classified in the AD group. All the patients who met the inclusion criteria were asked by the clinicians in the institutions to take part in the study. The study population consisted of 77 patients (mild AD: $n=36$; depression: $\mathrm{n}=41$ ) who were prospectively recruited in a memory clinic (AD: $n=30$; depression: $\mathrm{n}=2$ ) and in psychiatric hospitals (AD: $\mathrm{n}=6$; depression: $\mathrm{n}=39$ ).

The healthy controls were recruited from the general population (by contacting senior citizens' associations). The inclusion criteria of this healthy control group $(n=47)$ were: (1) no history of neurological or psychiatric disease, (2) no active psychiatric disorder, and (3) no organic diseases involving the central nervous system. This information was obtained by asking the subjects questions by means of a structured interview. Other inclusion criteria were: MMSE > 23 and Geriatric Depression Scale score $($ GDS $)$ (Yesavage, 1988) < 11, indicating the absence of severe cognitive and affective problems. The MMSE and GDS scores were both gathered by the researcher.

\section{Measures}

The MMSE, a short screening instrument for cognitive functions, the GDS, a scale to assess depressive symptoms, the 10-RAVLT, a 10-word-list verbal learning task, and the Raven Coloured Progressive Matrices (RCPM) (Raven, 1984) as a non-verbal interference task were administered to all subjects.

\section{$\underline{10-R A V L T}$}

The 10-RAVLT is a word-list learning task (10 unrelated words) consisting of 5 consecutive free recall trials and a recall (delayed recall) trial after an interference 
period. Additionally there is also a delayed recognition trial. The 10 nouns are read aloud, with a 2-seconds interval between each word, by the examiner, for 5 consecutive trials (trials 1 to 5), each trial followed by a free recall test. Patients are instructed that they will hear a list of 10 words and that they will be asked to repeat as many words as possible. The order, in which the words are presented, remains fixed across trials. Instructions are repeated before each trial in order to minimize forgetting. After a 20-minute delay period, in which non-verbal activities are carried out (in this study the RCPM is administered), each subject is again asked to recall as many words as possible (delayed recall trial). In the delayed recognition trial, patients have to point out whether the given word was in the original list or not (10 target words are presented among 10 distracters). Hits and correct rejections are recorded.

\section{$\underline{M M S E}$}

The MMSE is a structured test used to assess general cognitive status in an older adult population with good psychometric properties (Folstein, Folstein, \& McHugh, 1975).

\section{$\underline{G D S}$}

The GDS, consisting of 30 items, was developed as a basic screening measure for depression in older adults. The items should be answered by 'yes' or 'no'. A score of 11 or lower is the threshold separating depressed patients $(\geq 11)$ from non-depressed patients (<11) (Jongenelis et al., 2005).

\section{Procedure}

The study was approved by the local ethics committees. Written informed consent was obtained from all subjects. 
After a diagnosis was made by the clinicians in the institutions, the test instructor (all subjects were tested by the same research psychologist), who was not blinded for the clinical diagnosis, administered the MMSE, the GDS, the 10-RAVLT, and the RCPM to all subjects. These tests were part of a larger research test battery that took $+/-60$ minutes to administer. For this study, only the results of the MMSE, the GDS and the 10-RAVLT were used. For a detailed description of the other measures we refer to Dierckx et al. (2007) and Dierckx et al. (2008).

To ascertain whether depressed patients obtain higher scores on recognition, the delayed recognition task was used (a maximum score of 20 could be obtained: 10 for the hits and 10 for the correct rejections). To check the hypothesis that rate of forgetting is higher among $\mathrm{AD}$ patients than among depressed elderly patients, a percentage of forgetting was calculated as follows " $100 \mathrm{x}$ difference between the number of words correctly recalled after the fifth reading and at the delayed recall test / number of words correctly recalled during the fifth trial" (see Estévez-Gonzalez et al., 2003).

\section{Statistical analyses}

Intergroup differences in age, years of education, delayed recognition scores and percentage of forgetting were examined using one way analysis of variance (ANOVA). If significant main effects were shown, Scheffé post hoc analyses were performed. Intergroup differences in gender and use of benzodiazepines, antidepressants and antipsychotics were analyzed using chi-square tests. A criterion alpha level of .05 was used throughout the analyses.

Receiver Operating Characteristic curves (ROC curves) were drawn to determine the diagnostic accuracy of recognition and rate of forgetting in discriminating AD patients 
from depressed patients. When an Area Under the ROC Curve (AUC) of more than .80 was achieved an ideal cut off point with the highest sensitivity (S) and specificity (Sp) was determined. Afterwards $\mathrm{S}$ and $\mathrm{Sp}$ were calculated. As the meaning of a score on a test and its positive and negative predictive power (PPV, NPV) depend not only on the test's psychometric properties, such as sensitivity and specificity, but also on the rate of the disorder within the sample (Streiner, 2003), Positive and negative predictive values were calculated according to Bayes' theorem (Elwood, 1993), in which the prevalence was taken into account. Positive and negative predictive value data are then presented for various AD base rates.

A discriminant function analysis was performed to determine how well we could predict to which group a particular subject in our population belonged by using respectively the recognition and percentage of forgetting score. In order to check our assumption that percentage of forgetting scores might be more accurate for the early differentiation between $\mathrm{AD}$ and depression than delayed free recall, a discriminant analysis was also performed with delayed free recall as independent variable. Percentages of correctly (and incorrectly) identified mild AD and depressed elderly patients were obtained.

Data were analyzed using SPSS for Windows 16.0.

\section{Results}

\section{Demographic variables}

Table 1 lists the descriptive variables of the 3 groups with respect to age, gender and years of education.

A one-way ANOVA could not demonstrate significant differences in age $(F(2,121)=$ $1.91, p=.152)$ or number of years of education $(F(2,121)=2.85, p=.062)$ between the different diagnostic categories. 
A chi square analysis revealed differences in gender distribution between the 3 diagnostic groups $\left(\operatorname{chi}^{2}(2)=12.88, p=.002\right)$. The depression group contained more women than the $\mathrm{AD}\left(c h i^{2}(1)=4.76, p=.036\right)$ and healthy control groups $\left(c h i^{2}(1)=\right.$ 12.93, $p=.0001$ ). However, a MANOVA with (1) recognition and (2) percentage of forgetting as dependent variables and diagnosis and gender as independent variables could not demonstrate a significant interaction effect of diagnosis and gender ( $F$ (4, $236)=1.73, p=.144)$, nor a main effect of gender $(F(2,117)=0.66, p=.517)$.

As expected GDS-scores (i.e. the amount of depressive symptoms) were higher among depressed patients than among mild AD patients and healthy control subjects. However, a MANCOVA with (1) recognition and (2) percentage of forgetting as dependent variables, diagnosis as independent variable and score on the GDS as a covariate could not demonstrate a significant interaction effect of diagnosis and GDS score $(F(4,234)=0.31, p=.869)$, nor a main effect of GDS score (i.e. amount of depressive symptoms $)(F(2,116)=1.13, p=.625)$.

In our study, we used a naturalistic sample of elderly subjects who received a variety of pharmacological treatments. Chi square analyses demonstrated that in the depression group more subjects were taking benzodiazepines and antidepressants respectively than in the $\mathrm{AD}\left(\operatorname{chi}^{2}(1)=22.01, p=.0001 ; \operatorname{chi}^{2}(1)=25.92, p=.0001\right)$, and healthy control group $\left(\operatorname{chi}^{2}(1)=17.84, p=.0001 ; \operatorname{chi}^{2}(1)=63.46, p=.0001\right)$. No significant differences in use of benzodiazepines were found between subjects in the $\mathrm{AD}$ and healthy control group $\left(\operatorname{chi}^{2}(1)=0.98, p=.371\right)$. However, there were more subjects in the $\operatorname{AD}\left(\operatorname{chi}^{2}(1)=12.90, p=.0001\right)$ group who were using antidepressant medication as compared to the healthy control group. A MANOVA with (1) recognition and (2) percentage of forgetting as dependent variables and respectively (1) diagnosis and benzodiazepine use and (2) diagnosis and antidepressants as 
independent variables could not demonstrate a significant interaction effect of diagnosis and use of benzodiazepines $(F(4,198)=1.26, p=.288)$, nor an interaction effect of diagnosis and use of antidepressants benzodiazepines $(F(4,198)=1.99, p=$ $.097)$, nor a main effect of use of benzodiazepines $(F(2,99)=0.15, p=.863)$ and antidepressants $(F(2,99)=1.04, p=.356)$.

As far as use of antipsychotic medication is concerned no differences were found between $\mathrm{AD}$ and depressed patients $\left(c h i^{2}(1)=0.92, p=.437\right)$. More $\mathrm{AD}$ and respectively more depressed patients were taking antipsychotic medication than healthy control subjects $\left(\operatorname{chi}^{2}(1)=12.73, p=.0001 ; \operatorname{chi}^{2}(1)=18.96, p=.0001\right)$.

Twenty three (out of 36) mild AD patients were taking cholinesterase-inhibitors.

In summary: all depressed patients $(n=41)$ were taking some kind of psychotropic medication, as was also the case with most AD patients (28 out of 36). Only 10 (out of 47) healthy controls were receiving psychotropic pharmacological treatment.

\section{Recognition}

A one-way ANOVA $(F(2,121)=37.61, p=.0001)$ and post-hoc Scheffé tests showed that $\mathrm{AD}$ patients had significantly lower sores on the delayed recognition task than depressed elderly patients $(p=.0001)$ and healthy controls $(p=.0001)$. No significant differences were found between depressed elderly patients and healthy controls $(p=.423)$.

When the total score of delayed recognition was distributed into hits and correct rejections, a one-way ANOVA with post hoc Scheffé demonstrated no significant differences in the total amount of hits between the mild AD and depressed patients ( $p$ $=.793)$. The differences in delayed recognition mentioned above are consequently mainly due to significant differences in correct rejections $(F(2,121)=30.53, p=$ .0001), whereby AD patients had significantly lower sores on the amount of correct 
rejections than depressed elderly patients $(p=.0001)$ and healthy controls $(p=.0001)$. No significant differences were found between depressed patients and healthy control subjects $(p=.924)$.

\section{Rate of forgetting}

According to a one-way ANOVA, the percentage of forgetting was significantly different between the 3 diagnostic groups $(F(2,121)=38.25, p=.0001)$. Post hoc Scheffé tests showed that AD patients had a significantly $(p=.0001)$ higher percentage of forgetting than depressed patients $(p=.0001)$ and healthy controls $(p=.0001)$. The percentage of forgetting of depressed patients and healthy control subjects was not significantly different $(p=.894)$.

In the differentiation between mild $\mathrm{AD}$ and depressed patients, for both recognition and percentage of forgetting scores, a statistical power of more than .80 was obtained. See table 2 for an overview of the mean MMSE, GDS, delayed recognition and percentage of forgetting scores.

\section{ROC-curves}

\section{Differentiating AD patients from depressed elderly}

The AUC indicated that the delayed recognition $(\mathrm{AUC}=.83, p=.0001)$ and percentage of forgetting (AUC $=.87, p=.0001$ ) would both be considered to be good in differentiating AD patients from depressed elderly patients. See Fig. 1 and 2 for the ROC curves.

For delayed recognition the optimal cut off point was $18 / 20$ (sensitivity $=78 \%$, specificity $=73 \%$ ) whereas for percentage of forgetting, the optimal cut off was determined at $60 \%$ (sensitivity $=81 \%$, specificity $=90 \%)$. 
Predictive power, as measured by the positive and negative predictive values, is presented in tables 3 and 4.

\section{Discussion}

\section{Recognition}

It has been shown that impairment of acquisition and consolidation of episodic memory traces is the earliest sign of the underlying degenerative process of AD (Perry et al., 2000; Au, Chan, \& Chiu, 2003), whereas depressed patients may demonstrate essentially normal memory consolidation (Shankle et al., 2005). As delayed recognition measures whether or not the word has been encoded and consolidated, we hypothesized that recognition would be a useful criterion to differentiate between subjects with mild AD and depressed patients. Indeed, the delayed recognition score of the 10-RAVLT was significantly different between AD patients and depressed elderly patients, with AD patients obtaining lower scores than depressed patients. The suggested absence of consolidation deficits among depressed patients together with the fact that recognition is not cognitively effortful (Fossati et al., 2004), so that the effects of impaired attention, inefficient strategies, motivation problems, or lack of initiative are minimized (Kausler, 1994), may account for good performance on delayed recognition among depressed patients, whereas among AD patients poor performance was obtained due to consolidation deficits.

We also demonstrated that AD patients and depressed elderly patients differed only on their correct rejection scores, as the hit score was not significantly different between $\mathrm{AD}$ and depressed patients. This is probably due to the presence of a liberal bias among $\mathrm{AD}$ patients, whereby recognition is marked by an increased amount of false positives (Burt, Zembar, \& Niederehe, 1995). 
Generally speaking, our study revealed that delayed recognition of the 10-RAVLT may be a useful criterion on the basis of which to differentiate AD patients from depressed elderly patients. Indeed, the ROC curve showed that diagnostic accuracy was good $(\mathrm{AUC}=.83)$; with a cut-off point of 18 out of 20 , a sensitivity of $78 \%$ and a specificity of $73 \%$ were obtained.

\section{Rates of forgetting}

Our study showed that $\mathrm{AD}$ patients could be differentiated from depressed elderly patients on the basis of the percentage of forgetting. AD patients showed a higher percentage of forgetting than depressed patients. A diagnostic accuracy of .87 was obtained, which can be considered good as it exceeds the minimal threshold of $80 \%$ defined in the generally accepted criteria for diagnostic biological markers for AD (The Ronald and Nancy Reagan Research Institute of the Alzheimer's Association and the National Institute on Aging Working Group, 1998). With a cut-off point of $60 \%$, a sensitivity of $81 \%$ and a specificity of $90 \%$ were obtained. In AD patients, it has been shown that information is rapidly lost from episodic memory, indicating a failure to consolidate information effectively (Hart, Kwentus, Taylor, \& Harkins, 1987; Perry et al., 2000; Kopelman, 1992).

As can be seen from table 5, the percentage of forgetting was a better operationalization of the rate of forgetting than delayed free recall: according to percentage of forgetting $90 \%$ of depressed patients were classified correctly, whereas on delayed free recall only $71 \%$ of depressed patients were correctly classified. With the more traditional method (i.e. delayed free recall), more depressed patients were thought to have mild AD. As it is known that depressed patients learn fewer words (e.g. Gainotti \& Marra, 1994), our results support the hypothesis that it is better to operationalize the rate of forgetting on the basis of the percentage of forgetting, in 
which the amount of initially learned information is taken into account, rather than on the basis of delayed free recall.

\section{General discussion}

In a previous study (Dierckx et al., 2007), we found that cued recall, operationalized by the combined scores on the Visual Association Test (Lindeboom et al., 2002) and on the Memory Impairment Screen-plus (Buschke et al., 1999), is a criterion that is useful in differentiating AD patients from depressed and healthy individuals. These cued recall tests therefore appeared to be very useful instruments for the operationalization of the new research criteria for the diagnosis of AD as proposed by Dubois et al. (2007), as these authors suggest that the core diagnostic criterion is the presence of an episodic memory impairment that consists of a recall deficit that does not normalize or improve significantly after cueing.

However, this study revealed that the 10-RAVLT (a very widely and frequently used verbal learning test) may also be a useful instrument for the early detection of $\mathrm{AD}$, especially when it comes to making an early differentiation between, on the one hand, mild AD and, on the other hand, depression and healthy aging among elderly subjects. Both delayed recognition and the percentage of forgetting, in our study, show a diagnostic accuracy (high sensitivity and specificity scores) that can be considered as useful criterion for differentiating between $\mathrm{AD}$ patients and depressed elderly patients and healthy controls. This is probably due to episodic memory consolidation problems, which are present in $\mathrm{AD}$ but are considered to be absent in depressed subjects and healthy aging subjects. However, these results are preliminary and need to be validated using alternate samples that include a higher number of subjects.A possible limitation of the present study is that we have no information of the patients who did meet the inclusion criteria but did not want to participate in the study. It may 
be that the depressed patients who refused were more severely depressed. However, most depressed patients $(n=39)$ scored $\geq 11 / 30$ on the GDS, indicating the presence of significant depressive symptoms. Moreover, 21 patients obtained a score of more than 20/30, indicating severe depression. We can thus assume that severe depression did not lead to a selection bias through the refusal to participate.

A further limitation is that the psychologist who administered the measures to the subjects was not blinded to the clinical diagnosis. However, as all the measurements require standard administration procedures, possible results biases are minimal.

Another possible limitation of the present study is that we used a naturalistic sample of elderly subjects who typically receive a variety of pharmacological treatments, rather than highly controlled clinical research subjects. In general, benzodiazepine use was more frequent among depressed elderly patients than among $\mathrm{AD}$ patients and healthy controls. Since it is well documented that benzodiazepines have a negative effect on cognition (Perna, 2004), this could have had an effect on delayed recognition and the percentage of forgetting scores. However, we obtained neither a significant interaction effect of group and benzodiazepine use nor a main effect of benzodiazepine use.

In our study, 23 out of the $35 \mathrm{AD}$ patients and none of the depressed elderly patients were taking cholinesterase inhibitor medication. Because a recent study (Crowell, Paramadevan, Abdullah, \& Mullan, 2006) showed that this medication may have a beneficial effect on the recognition memory performance of $\mathrm{AD}$ patients, the use of cholinesterase inhibitors may have accounted for the slightly lower accuracy scores obtained by delayed recognition compared to the percentage of forgetting in differentiating $\mathrm{AD}$ from depressed patients.

Furthermore, as expected, antidepressant use was more frequent among depressed patients. However, most of the patients were treated with serotonin reuptake 
inhibitors, and some were receiving low-dose tricyclic antidepressant medication, minimizing the potential cognitive side effects. A significant interaction effect of diagnosis and use of antidepressants was found on the recognition task. Since there is some evidence to suggest that antidepressant medication might have neuroprotective properties, particularly in the hippocampus (Haynes, Barber, \& Mitchell, 2004), the performance of the depressed patients on the recognition score may have been positively influenced by the use of antidepressant medication. However, it has been argued that cognitive improvement following pharmacological treatment with antidepressants is seen only after an improvement in depressive symptoms (Doraiswamy et al., 2003). In our study, all the depressed patients met the diagnostic criteria for major depressive disorder during the study. Moreover, delayed free recall is considered to be the memory measurement with the greatest sensitivity for the early detection of AD (Swainson et al., 2001), since it is anatomically related to the medial temporal lobe (Campo, Morales, \& Martinez-Castillo, 2003). In our study, the percentage of forgetting, which is derived from the delayed free recall score, appeared not to be influenced by the use of antidepressants. We therefore assume that the influence of antidepressants on our test scores is minimal. However, to check this assumption future studies must be carried out with treatment-naïve patients.

\section{Acknowledgements}

This research was supported by the Research Fund of the Vrije Universiteit Brussel (OZR) and the Special Research Fund of the University of Antwerp, Stichting Alzheimer Onderzoek, the Thomas Riellaerts Research Fund, the Institute BornBunge, the Agreement between the Institute Born-Bunge and the University of Antwerp, Medical Research Foundation Antwerp, Neurosearch Antwerp, the Research Foundation - Flanders (FWO-F), the Institute for Promotion of Innovation 
through Science and Technology in Flanders (IWT-Vlaanderen) and the InterUniversity Attraction Poles (IAP) program P6/43 of the Belgian Federal Science Policy office (BELSPO).

We would like to thank Karen Maertens and Michael Van Buggenhout for assistance in the recruitment of the study population and the following psychiatric institutions for giving the opportunity of testing the patients: Broeders Alexianen Tienen, SintNorbertus Duffel, Sint-Jozef Pittem and Sint-Jozef Kortenberg.

\section{Conflict of interest}

None. 


\section{References}

American Psychiatric Association (1994). Diagnostic and Statistical Manual of Mental Disorders. (4th ed.) Washington: American Psychiatric Association.

Au, A., Chan, A. S., \& Chiu, H. (2003). Verbal learning in Alzheimer's dementia. Journal of the International Neuropsychological Society, 9, 363-375.

Bearden, C. E., Glahn, D. C., Monkul, E. S., Barrett, J., Najt, P., Villarreal, V. et al. (2006). Patterns of memory impairment in bipolar disorder and unipolar major depression. Psychiatry Research, 142, 139-150.

Burt, D. B., Zembar, M. J., \& Niederehe, G. (1995). Depression and Memory Impairment - A Metaanalysis of the Association, Its Pattern, and Specificity. Psychological Bulletin, 117, 285-305.

Buschke, H., Kuslansky, G., Katz, M., Stewart, W. F., Sliwinski, M. J., Eckholdt, H. M., \& Lipton, R. B. (1999). Screening for dementia with the memory impairment screen. Neurology, 52, 231-238.

Campo,P., Morales,M. \& Martinez-Castillo,E. (2003). Discrimination of normal from demented elderly on a Spanish version of the verbal Selective Reminding Test. Journal of Clinical and Experimental Neuropsychology, 25, 991999.

Crowell, T. A., Paramadevan, J., Abdullah, L., \& Mullan, M. (2006). Beneficial effect of cholinesterase inhibitor medications on recognition memory performance in mild to moderate Alzheimer's disease: Preliminary findings. Journal of Geriatric Psychiatry and Neurology, 19, 13-15. 
Dierckx, E., Engelborghs, S., De Raedt, R., De Deyn, P. P., and PonjaertKristoffersen, I. (2007). Differentiation between Mild Cognitive Impairment, Alzheimer's disease and depression by means of cued recall. Psychological Medicine, $37,747-755$.

Dierckx, E., Engelborghs, S., De Raedt, R., Van Buggenhout, M., De Deyn, P.P., Verleye, G., and Ponjaert-Kristoffersen, I. (2008). Differentiation between dementia and depression: Can the difference between actual and premorbid intelligence be useful? Journal of Geriatric Psychiatry and Neurology, 21, 242-249.

Doraiswamy, P.M., Krishnan, K.R.R., Oxman, T., Jenkyn, L.R., Coffey, D.J., Burt, T. \& Clary, C.M. (2003). Does antidepressant therapy improve cognition in elderly depressed patients? Journal of gerontology, 12, 1137-1144.

Dubois, B., Feldman, H.H., Jacova, C., DeKosky, S. T., Barberger-Gateau, P., Cummings, J., et al. (2007). Research criteria for the diagnosis of Alzheimer's disease: revising the NINCDS-ADRDA criteria. Lancet Neurology, 6, 734-746.

Elwood, R. (1993). Clinical discriminations and neuropsychological tests: an appeal to Bayes' theorem. Clinical Neuropsychologist, 7, 224-233.

Estevez-Gonzalez, A., Kulisevsky, J., Boltes, A., Otermin, P., \& GarciaSanchez, C. (2003). Rey verbal learning test is a useful tool for differential diagnosis in the preclinical phase of Alzheimer's disease: comparison with mild cognitive impairment and normal aging. International Journal of Geriatric Psychiatry, 18, 1021-1028. 
Folstein, M. F., Folstein, S. E., \& McHugh, P. R. (1975). Mini-Mental State Practical Method for Grading Cognitive State of Patients for Clinician. Journal of Psychiatric Research, 12, 189-198.

Fossati, P., Harvey, P. O., Le Bastard, G., Ergis, A. M., Jouvent, R., \& Allilaire, J. F. (2004). Verbal memory performance of patients with a first depressive episode and patients with unipolar and bipolar recurrent depression. Journal of Psychiatric Research, 38, 137-144.

Gainotti, G. \& Marra, C. (1994). Some Aspects of Memory Disorders Clearly Distinguish Dementia of the Alzheimers Type from Depressive Pseudo-Dementia. Journal of Clinical and Experimental Neuropsychology, 16, 65-78.

Hart, R. P., Kwentus, J. A., Taylor, J. R., \& Harkins, S. W. (1987). Rate of Forgetting in Dementia and Depression. Journal of Consulting and Clinical Psychology, 55, 101-105.

Haynes, L.E., Barber, D. \& Mitchell, I. J. (2004). Chronic antidepressant medication attenuates dexamethasone-induced neuronal death and sublethal neuronal damage in the hippocampus and striatum. Brain Research, 1026, 157-167.

Inouye, S. K., Vandyck, C. H., Alessi, C. A., Balkin, S., Siegal, A. P., \& Horwitz, R. I. (1990). Clarifying Confusion - the Confusion Assessment Method - A New Method for Detection of Delirium. Annals of Internal Medicine, 113, 941-948.

Jongenelis, K., Pot, A. M., Eisses, A. M. H., Gerritsen, D. L., Derksen, M., Beekman, A. T. F., Kluiter, H., \& Ribbe, M. W. (2005). Diagnostic accuracy of the original 30-item and shortened versions of the Geriatric Depression Scale in nursing home patients. International Journal of Geriatric Psychiatry, 20, $1067-1074$. 
Karrasch, M., Sinerva, E., Gronholm, P., Rinne, J., \& Laine, M. (2005). CERAD test performances in amnestic mild cognitive impairment and Alzheimer's disease. Acta Neurologica Scandinavica, 111, 172-179.

Kausler, D. H. (1994). Learning and memory in normal aging. San Diego: Academic Press.

Kopelman, M. D. (1992). Storage, forgetting, and retrieval in the anterograde and retrograde amnesia of Alzheimer dementia. In L.Backman (Ed.), (pp. 45-72). Amsterdam: Elsevier Science Publishers.

Lindeboom, J., Schmand, B., Tulner, L., Walstra, G., \& Jonker, C. (2002). Visual association test to detect early dementia of the Alzheimer type. Journal of Neurology Neurosurgery and Psychiatry, 73, 126-133.

MacDonald, S. W. S., Stigsdotter-Neely, A., Derwinger, A., \& Backman, L. (2006). Rate of acquisition, adult age, and basic cognitive abilities predict forgetting: New views on a classic problem. Journal of Experimental Psychology-General, 135, 368-390.

McKhann, G., Drachman, D., Folstein, M., Katzman, R., Price, D., \& Stadlan, E. M. (1984). Clinical-Diagnosis of Alzheimers-Disease - Report of the Nincds-Adrda Work Group Under the Auspices of Department-Of-Health-And-Human-Services Task-Force on Alzheimers-Disease. Neurology, 34, 939-944.

Perna, R. (2004). Benzodiazepines and antipsychotics - Cognitive side effects. Journal of Head Trauma Rehabilitation, 19, 516-518. 
Perry, R. J., Watson, P., \& Hodges, J. R. (2000). The nature and staging of attention dysfunction in early (minimal and mild) Alzheimer's disease: relationship to episodic and semantic memory impairment. Neuropsychologia, 38, 252-271.

Ravaglia, G., Forti, P., Maioli, F., Martelli, M., Servadei, L., Brunetti, N., et al. (2006). Conversion of mild cognitive impairment to dementia: Predictive role of mild cognitive impairment subtypes and vascular risk factors. Dementia and Geriatric Cognitive Disorders, 21, 51-58.

Raven, J. C. (1984). Manual for the Coloured Progressive Matrices (Revised). Windsor, UK: NFER-Nelson.

Shankle, W. R., Romney, A. K., Hara, J., Fortier, D., Dick, M. B., Chen, J. M. et al. (2005). Methods to improve the detection of mild cognitive impairment. Proceedings of the National Academy of Sciences of the United States of America, $102,4919-4924$.

Sonkusare, S. K., Kaul, C. L., \& Ramarao, P. (2005). Dementia of Alzheimer's disease and other neurodegenerative disorders - memantine, a new hope. Pharmacological Research, 51, 1-17.

Streiner, D. L. (2003). Diagnosing tests: using and misusing diagnostic and screening tests. Journal of personality assessment, 81, 209-219.

Swainson, R., Hodges, J. R., Galton, C. J., Semple, J., Michael, A., Dunn, B. D. et al. (2001). Early detection and differential diagnosis of Alzheimer's disease and depression with neuropsychological tasks. Dementia and Geriatric Cognitive Disorders, 12, 265-280. 
The Ronald and Nancy Reagan Research Institute of the Alzheimer's Association and the National Institute on Aging Working Group. (1998). Consensus Report of the Working Group on Molecular and Biochemical Markers of Alzheimer's Disease. Neurobiology of aging, 19, 109-116.

Tuokko, H. \& Hadjistavropoulos, T. (1998). An assessment guide to geriatric neuropsychology. New Jersey: Laurence Erlbaum associates.

VVKP Werkgroep Klinische gerontopsychologie (1998). Handleiding Vlaamse Dementiebatterij-2. Leuven.

Yesavage, J. A. (1988). Geriatric Depression Scale. Psychopharmacology Bulletin, 24, 709-711. 
Table 1. Sample description: demographic variables gender, age and years of education

\begin{tabular}{llll}
\hline & Mild AD & Depression & Controls \\
\hline $\mathrm{N}$ & 36 & 41 & 47 \\
\hline Male/female & $13 / 23$ & $6 / 35$ & $24 / 23$ \\
\hline Mean (SD) age & $76.6(6.0)$ & $74.1(5.2)$ & $75.7(5.9)$ \\
in years & & & \\
\hline Mean (SD) years & $10.2(2.5)$ & $10.4(2.5)$ & $11.6(3.4)$ \\
of education & & & \\
\hline
\end{tabular}

